Heine-Jahrbuch 
Herausgegeben in Verbindung mit der Heinrich-Heine-Gesellschaft 


\title{
HEINE-JAHRBUCH 2002
}

\author{
4I. Jahrgang \\ Herausgegeben von Joseph A. Kruse \\ Heinrich-Heine-Institut \\ der Landeshauptstadt Düsseldorf
}

Verlag J. B. Metzler

Stuttgart - Weimar 


$$
\begin{gathered}
\text { Anschrift des Herausgebers: } \\
\text { Joseph A. Kruse } \\
\text { Heinrich-Heine-Institut }
\end{gathered}
$$

Bilker Straße I2-I4, 40213 Düsseldorf

Redaktion: Karin Füllner und Marianne Tilch

Die Deutsche Bibliothek - CIP Einheitsaufnahme

\author{
Heine-Jahrbuch ... / hrsg. in Verbindung mit der Heinrich-Heine- \\ Gesellschaft. -Stuttgart ; Weimar : Metzler. \\ Erscheint jährl. - Früher im Verl. Hofmann und Campe, Hamburg.- \\ Aufnahme nach Jg. 34. 1995 \\ Darin aufgegangen: Heinrich-Heine-Gesellschaft, Düsseldorf
}

Jg. 34. 1995 - Verl.-Wechsel-Anzeige

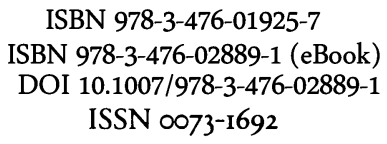

Dieses Werk einschließlich aller seiner Teile ist urheberrechtlich geschützt. Jede Verwertung außerhalb der engen Grenzen des Urheberrechtsgesetzes ist ohne Zustimmung des Verlages unzulässig und strafbar. Das gilt insbesondere für Vervielfältigungen, Übersetzungen, Mikroverfilmungen und die Einspeicherung und Verarbeitung in elektronischen Systemen.

(C) 2002 Springer-Verlag GmbH Deutschland Ursprünglich erschienen bei J.B. Metzlersche Verlagsbuchhandlung und Carl Ernst Poeschel Verlag GmbH in Stuttgart 2002

www.metzlerverlag.de Info@metzlerverlag.de 


\section{Inhalt}

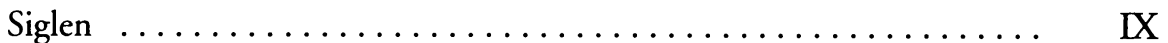

Aufsätze

I.

Stefan Neuhaus · Dekonstruktion nationaler Mythologeme:

Heinrich Heine und Deutschland $\ldots \ldots \ldots \ldots \ldots \ldots \ldots$ I

John Pizer · Heine's Unique Relationship to Goethe's Weltliteratur

Paradigm ................................. I8

Stuart Ferguson - Heinrich Heines "Die Bäder von Lukka" als

perverse Ethopoetik: Die Ästhetik der Sexualabweichung und/oder

die Rhetorik homophobischer Verunglimpfung ............ 37

Christine Mielke · Der Tod und das novellistische Erzählen.

Heinrich Heines "Florentinische Nächte" .............. 54

II.

Ralf Schnell • Heinrich Heine und Bertolt Brecht.

Das Exil als poetische Lebensform . . . . . . . . . . . . . . $8_{3}$

Karlheinz Fingerhut • "Manchmal nur, in dunkeln Zeiten".

Heine, Kafka, Celan. Schreibweisen jüdischer Selbstreflexion ...... 106

Liu Min - Heines Lyrik in China - vom Anfang bis $1949 \ldots \ldots \ldots \ldots . \quad$ I30

Inge Rippmann - Emanzipation und Akkulturation.

Ein nicht ganz typisches Beispiel: Ludwig Börne $\ldots \ldots \ldots \ldots \ldots \ldots$ I6I 
Kleinere Beiträge

Constanze Wachsmann • „Ein mutiger Trommler der Revolution«.

Zur Heinrich Heine-Rezeption in der Sowjetunion (1917-1953) . . . . I88

Ursula Broicher . Der legislatorische Sonderweg des Rheinlandes oder Heine und der Fonk-Prozess . . . . . . . . . . . . . . . 205

Horst Heidermann • I847: Ein "Anti-Musik-Verein» im Wohnhaus der

Familie Heine $\ldots \ldots \ldots \ldots \ldots \ldots \ldots \ldots \ldots \ldots \ldots \ldots \ldots$ 22I

Notiz

Wilhelm Gössmann · Heine beim Wort genommen:

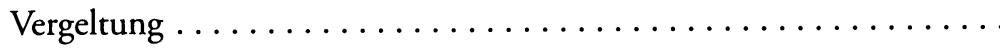

Bericht

Joseph A. Kruse . Heine in Jerusalem . . . . . . . . . . . . . . 229

Heinrich-Heine-Institut. Sammlungen und Bestände. Aus der Arbeit des Hauses. Ausstellungseröffungen

Jean-Pierre Lefebvre . Über Paul Celan in der rue d'Ulm . . . . . . . . . 233

Johannes Stüttgen • "Joseph Beuys. Texte" . . . . . . . . . . . . . 239

Karin Füllner · „Dieses ist die neue Welt!« Das Düsseldorfer

Studierenden-Kolloquium 200 I mit neuen Arbeiten über

Heinrich Heine ....................... 245

Nachruf

Wilhelm Gössmann · Nachruf auf Fritz Mende . . . . . . . . . . $\quad 248$ 
Buchbesprechungen

Wolfgang Beutin/Thomas Bütow/Johann Dvořák/Ludwig Fischer

(Hrsg.) • "Die Emanzipation des Volkes war die große Aufgabe unseres

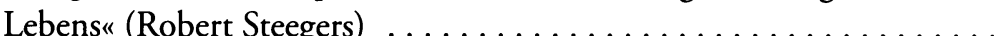

Klaus Briegleb/Itta Shedletzky (Hrsg.) · Das Jerusalemer Heine-Symposium. Gedächtnis, Mythos, Modernität (Sikander Singh) . . . . . .

Ege Forschungen zur deutschen Sprach- und Literaturwissenschaft Heinrich-Heine (Karin Füllner) $\ldots \ldots \ldots \ldots \ldots \ldots \ldots \ldots \ldots \ldots \ldots \ldots$

Bernd Füllner (Hrsg.) · Briefkultur im Vormärz. Vorträge der Tagung des Forum Vormärz Forschung und der Heinrich-Heine-Gesellschaft

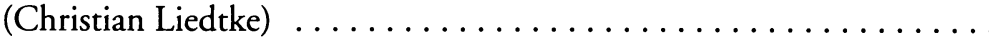

Jost Hermand/Robert C. Holub (Hrsg.) · Heinrich Heine’s Contested Identities. Politics, Religion, and Nationalism in Nineteenth-Century Germany. (Karin Sousa)

Olaf Hildebrand · Emanzipation und Versöhnung. Aspekte des Sensualismus im Werk Heinrich Heines unter besonderer Berücksichtigung der "Reisebilder" (Robert Steegers) . . . . . . . . . . . . . .

Julia Freifrau Hiller von Gärtringen/Detlev Hellfaier · Grabbe im Original. Autographen, Bilder, Dokumente (Joseph A. Kruse) . . . . . . . . .

Hans Hörling (Hrsg.) • Die französische Heine-Kritik. Bd. 2. I835-I845

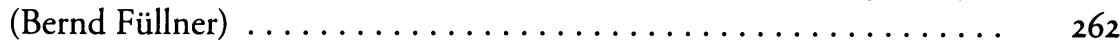

Karl-Josef Kuschel • „Gottes grausamer Spaß?» Heinrich Heines Leben mit der Katastrophe (Wilhelm Gössmann) . . . . . . . . . . . . . . 264

Hugh Powell (Hrsg.) • Berliner Don Quixote. Jg. I832/33. Red. Adolf Glassbrenner (Olaf Briese)

Terence James Reed/ Alexander Stillmark (Hrsg.) . Heine und die Welt-

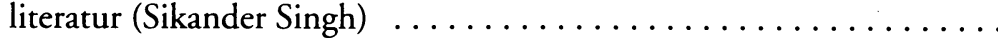

Mechthilde Vahsen - Die Politisierung des weiblichen Subjekts. Deutsche Romanautorinnen und die Französische Revolution I790-I820 (Ariane Neuhaus-Koch)

Heine-Literatur 2001/2002 mit Nachträgen . . . . . . . . . . . . . 
Preisaufgabe der Heinrich-Heine-Gesellschaft e. $V . \ldots \ldots \ldots \ldots \ldots \ldots$ 30I

Ankündigung des Düsseldorfer Studierenden-Kolloquiums 2003 . . . . . . . 302

Abbildungen .......................... 303

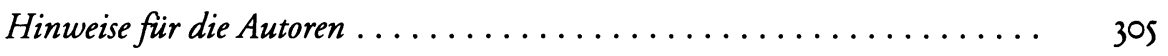

Mitarbeiter des Heine-Jahrbuchs 2002 . . . . . . . . . . . . . . . 307 


\title{
Siglen
}

\author{
I. H. Heine: Werke und Briefe
}

B = Heinrich Heine: Sämtliche Schriften. Hrsg. von Klaus Briegleb. München: Hanser 1968-I976, 6 Bände (6, II = Register)

DHA = Heinrich Heine: Historisch-kritische Gesamtausgabe der Werke. In Verbindung mit dem Heinrich-Heine-Institut hrsg. von Manfred Windfuhr. Hamburg: Hoffmann und Campe 1973-1997, I6 Bände

HSA = Heinrich Heine: Werke, Briefwechsel, Lebenszeugnisse. Säkularausgabe. Hrsg. von den Nationalen Forschungs- und Gedenkstätten der klassischen deutschen Literatur in Weimar (seit 1991: Stiftung Weimarer Klassik) und dem Centre National de la Recherche Scientifique in Paris. Berlin und Paris: Akademie und Editions du CNRS $1970 \mathrm{ff}$.

\section{Weitere Abkürzungen}

Galley/Estermann = Eberhard Galley und Alfred Estermann (Hrsg.): Heinrich Heines Werk im Urteil seiner Zeitgenossen. Hamburg: Hoffmann und Campe $1981 \mathrm{ff}$.

$\mathrm{HJb} \quad$ = Heine-Jahrbuch. Hrsg. vom Heinrich-Heine-Institut Düsseldorf. Hamburg: Hoffmann und Campe 1962-1994; Stuttgart: Metzler 1995 ff.

Mende $\quad=$ Fritz Mende: Heinrich Heine. Chronik seines Lebens und Werkes. Berlin: Akademie ${ }^{\mathrm{I}} \mathrm{1970} ;{ }^{2} \mathrm{I} 98 \mathrm{I}$

Seifert $\quad=$ Siegfried Seifert: Heine-Bibliographie 1954-1964. Berlin und Weimar: Aufbau 1968

Seifert/Volgina = Siegfried Seifert und Albina A. Volgina: Heine-Bibliographie 1965-1982. Berlin und Weimar (Hrsg.): Begegnungen mit Heine. Berichte der Zeitgenossen. Hamburg: Hoffmann und Campe 1973, 2 Bände

Wilamowitz = Erdmann von Wilamowitz-Moellendorf und Günther Mühlpfordt $(\dagger)$ : HeineBibliographie 1983-1995. Stuttgart und Weimar: Metzler 1998

Wilhelm/Galley = Gottfried Wilhelm und Eberhard Galley: Heine-Bibliographie [bis 1953]. Weimar: Arion 1960, 2 Bände 